\title{
Long-Lasting Primed State in Maize Plants: Salicylic Acid and Steroid Signaling Pathways as Key Players in the Early Activation of Immune Responses in Silks
}

\author{
Romina B Agostini, ${ }^{1}$ Agustina Postigo, ${ }^{1}$ Sebastian P. Rius, ${ }^{1}$ Gabriel E. Rech, ${ }^{2}$ \\ Valeria A. Campos-Bermudez, ${ }^{1,+}$ and Walter A. Vargas ${ }^{1,+}$ \\ ${ }^{1}$ Centro de Estudios Fotosintéticos y Bioquímicos (CEFOBI-CONICET), Universidad Nacional de Rosario, Suipacha 531, \\ S2002LRK, Argentina; and ${ }^{2}$ Institute of Evolutionary Biology (CSIC-Universitat Pompeu Fabra), Passeig Marítim de la \\ Barceloneta 37-49, 08003, Barcelona, Spain
}

Accepted 18 September 2018.

\begin{abstract}
In the present study, we investigated the induced systemic resistance (ISR) activated by the beneficial fungus Trichoderma atroviride in maize plants, and the early immunological responses triggered after challenge with the ear rot pathogen Fusarium verticillioides. By transcriptional analysis, we were able to identify the gene core set specifically modulated in silks of maize plants expressing ISR. Our results showed that the main transcriptional reprogramming falls into genes involved in five main functional categories: cell structure or cell wall, amino acid and protein metabolism, stress responses, signaling, and transport. Among these ISR-related genes, it is important to highlight novel findings regarding hormone metabolism and signaling. The expression of hormone-dependent genes was in good agreement with the abscisic acid, jasmonic acid, and salicylic acid (SA) levels detected in the plants under study. The experimental design allowed the identification of novel regulatory elements related to a heightened state of defense in silks and suggests that steroids and SA are central components of a master regulatory network controlling the immunity of silks during ISR. The results presented also provide evidence about the molecular mechanisms used by maize silks against $\boldsymbol{F}$. verticillioides to counteract pathogenic development and host invasion, including pathogenesis-related genes, plant cell-wall reinforcement, fungal cell-wall-degrading enzymes and secondary metabolism.
\end{abstract}

Pathogenic diseases of maize crop have a strong impact on the global economy, especially in areas where this cereal is a

Expression data have been deposited in the National Center for Biotechnology Information Gene Expression Omnibus under accession number GSE93760.

${ }^{\dagger}$ Corresponding authors: Walter A. Vargas;

E-mail: walter.a.vargas@ypftecnologia.com; and

Valeria A. Campos Bermudez; E-mail: campos@cefobi-conicet.gov.ar

Funding: Financial support was provided by Agencia Nacional de Promoción Científica y Tecnológica (ANPCyT) (PICT2013-1521 and PICT2014-0801), CONICET (PIP 0028), and Agencia Santafesina de Ciencia, Tecnología e Innovación.

*The $\boldsymbol{e}$-Xtra logo stands for "electronic extra" and indicates that three supplementary figures, three supplementary tables, and one supplementary text are published online.

(c) 2019 The American Phytopathological Society primary food commodity. The fungus Fusarium verticillioides (Sacc.) Nirenberg (synonym F. moniliforme J. Sheld., teleomorph Gibberella moniliformis Wineland), which is endemic in maize fields at harvest, is the main causal agent of maize ear rot (Battilani et al. 2008; Bottalico 1998; Chulze et al. 1996; Folcher et al. 2009; Logrieco et al. 2002). This pathogen can cause not only ear rotting but also produce massive amounts of mycotoxins belonging to the fumonisin family, which have deleterious effects on humans and farm animals.

At the silking stage, $F$. verticillioides invades the ear through the silk channel and colonizes the developing kernels via a narrow opening called the stylar canal (Duncan and Howard 2010; Koehler 1942). As colonization continues, the host plant triggers several defense mechanisms in an attempt to stop the infection process (Ferrigo et al. 2014; Lanubile et al. 2012, 2014).

Because no maize line displaying full resistance to ear rot disease has yet been identified, breeding may be the safest and most sustainable strategy to reduce $F$. verticillioides incidence in maize fields (Clements et al. 2004; Eller et al. 2008; Lanubile et al. 2011, 2017; Munkvold 2003). A heightened state of resistance can be achieved as the outcome of the plant interaction with beneficial microorganisms that enhance immune responses and reduce pathogenic impact (Pieterse et al. 2014; Schlaeppi and Bulgarelli 2015). Many species of rhizobacteria and fungi have been identified as beneficial for plant growth and integrity (plant growth-promoting rhizobacteria and plant growth-promoting fungi, respectively) (Pieterse et al. 2014). Thus, the use of these microorganisms to further investigate plant immune responses may represent a valuable resource to expose hidden molecular mechanisms essential to reduce disease development.

In this context, the genus Trichoderma includes many nonpathogenic soilborne (free-living) filamentous fungal species able to colonize plant roots as avirulent symbionts (Harman et al. 2004). Several Trichoderma spp. have been proven to be antagonist agents able to control fungal pathogens, oomycetes, and nematodes (Druzhinina et al. 2011). In addition, members of this fungal genus have been demonstrated to be plant growth promoters, improving and maintaining soil productivity and, thus, providing important benefits to agriculture (Hermosa et al. 2013). Trichoderma spp. can also stimulate local and distant immune responses (induced systemic resistance [ISR]) to prevent future pathogenic attacks (Pieterse et al. 2014). Priming of plant defenses is marked by changes in transcriptional, metabolic, and epigenetic levels after stimulus perception (Conrath 2011; Mauch-Mani et al. 2017). Upon pathogenic attack, the host plant (under a primed state) is able to mount a quick or 
robust immune response that defines the postchallenge outcome of the plant-pathogen interaction. As a result of this enhanced response, plants can effectively ward off pathogenic infections or develop stress tolerance. Priming has also been reported to be durable throughout the plant's life cycle, and transmitted to the following plant generations in some sort of plant immunological memory manner (de Medeiros et al. 2017; Mauch-Mani et al. 2017).

The molecular and physiological mechanisms underlying the outcome of priming and ISR are currently viewed as the central strategies used by Trichoderma spp. to counteract disease development in distant tissues (Pieterse et al. 2014). However, the nature of the ISR activated by Trichoderma spp. is still under discussion. Several studies have shown that some Trichoderma strains are able to elicit ISR in a jasmonic acid (JA)- and ethylene (ET)-dependent form in leaves of dicot plants (ContrerasCornejo et al. 2011; Hermosa et al. 2012; Salas-Marina et al. 2011), whereas others have concluded that Trichoderma might trigger systemic acquired resistance (SAR)-like responses in a salicylic acid (SA)-dependent form, mainly because of the upregulation of pathogenesis-related (PR) genes (PR1, PR2, and PR5) (Hermosa et al. 2012; Mathys et al. 2012). Trichoderma-mediated induction of the SA, ET or JA pathways within a single plant seems to indicate a rather intricate mechanism for resistance activation. The connection between SAR and ISR pathways may depend on the nature of the pathogen and the infected tissue (Korolev et al. 2008; Pieterse et al. 2012).

Among the molecular mechanisms underlying the physiological changes induced by Trichoderma in plants, leucine-rich repeat (LRR) proteins and mitogen-activated protein kinase cascades have been shown to participate in the activation of the immune system (Shoresh et al. 2010). Further studies have shown that, in the Arabidopsis-Trichoderma-Pseudomonas tripartite system, the transcription factor AtMYB72 acts as a node for signal convergence during the signaling events responsible for ISR activation (Segarra et al. 2009). In addition, the redox-dependent regulator NONEXPRESSOR OF PR GENES 1 (NPR1) (known as a transcriptional coactivator of several PR genes in an SA-dependent mechanism) has also been involved in the JA/ET signaling events responsible for ISR activation in Arabidopsis during the interaction between plant

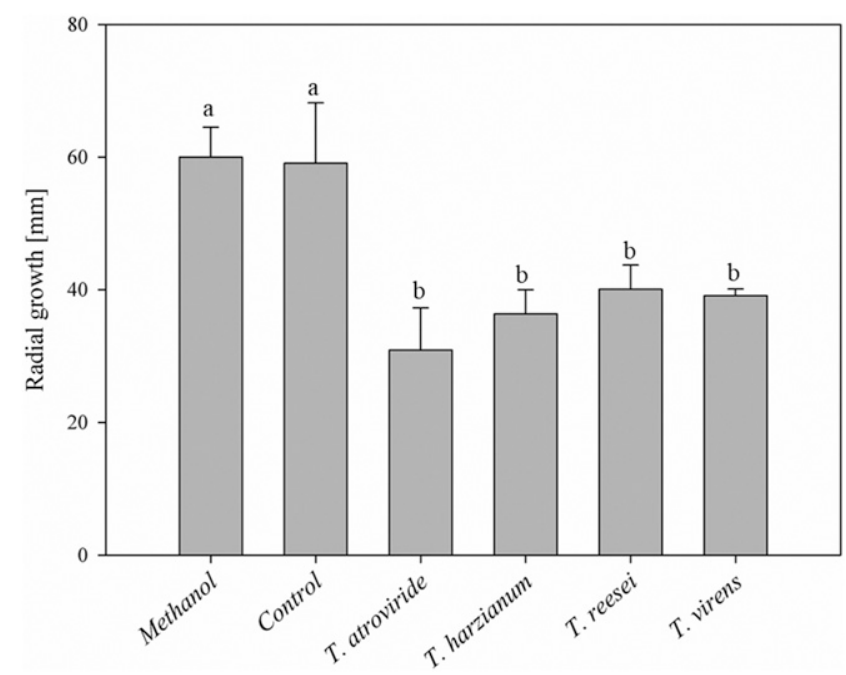

Fig. 1. Antimicrobial metabolites accumulated in maize leaves after Trichoderma root inoculation. Inhibition of the radial growth of Fusarium verticillioides in potato dextrose agar plates supplemented with methanolic extracts of leaves from control plants and plants inoculated with different strains of Trichoderma. Bars depict the mean value \pm standard deviation of three independent replicates. Bars with different letters differ significantly at a significance level of 5\% according to Tukey's test. growth-promoting rhizobacteria and plant growth-promoting fungi (Pieterse et al. 2012).

Several studies on the effects of Trichoderma on monocots have been performed using maize as the host plant. The study by Djonović et al. (2007), for example, described that ISR-like responses triggered by Trichoderma virens in maize plants involves mainly JA/ET and green leaf volatile signaling events. On the other hand, Ferrigo et al. (2014) demonstrated that inoculation of maize roots with $T$. harzianum consistently reduces the disease symptoms caused by $F$. verticillioides. Gene expression studies have shown that SA and JA/ET are key players in systemic immunity, and suggested that the heightened immune responses are due to an improved ability of the plant to detect the fungal pathogen. Similar results have also been reported by Saravanakumar et al. (2016), who demonstrated that T. harzianum is able to locally activate the maize root defense system by releasing extracellular cellulases, a phenomenon that is communicated to systemic tissues through JA/ET signaling events and results in a reduction of curvularia leaf spot disease.

Due to the lack of comprehensive studies related to ISR and disease resistance in maize, in this work, we sought to further investigate the transcriptional reprogramming in silk tissues from maize plants inoculated with Trichoderma spp. and challenged with $F$. verticillioides. The main goal of our research was to focus on early events ( $24 \mathrm{~h}$ after infection) of defense activation in plants subjected to priming during the whole crop cycle (approximately 2 months).

This study allowed the evaluation of the cellular reprogramming and regulatory events related to ISR in maize silks to counteract ear infection by $F$. verticillioides. Overall, the dataset obtained also provides critical information for a better understanding of hormonal and metabolic signals in distinct tissues from maize plants.

\section{RESULTS}

Maize inbred line B73/T. atroviride/F. verticilliodes as the model study system.

It is well-known that different species of Trichoderma can induce varying intensities of the host plant immune response. As mentioned above, in this study, we evaluated the systemic responses induced in maize by four reference strains of Trichoderma (T. atroviride IMI206040, T. harzianum, T. reesei Q6, and T. virens Gv-29-8). Before the evaluation of immune responses, Trichoderma spp. were confirmed to be confined to plant roots and, thus, any differential effect detected in leaves would be the result of the systemic responses induced after root colonization (Supplementary Fig. S1).

The first approach was to determine the synthesis of antifungal compounds in leaves in Trichoderma-root-inoculated maize plants. In this context, the $F$. verticillioides growth rate was evaluated on potato dextrose agar (PDA) plates in the presence of methanolic extracts prepared from maize plants. Colonies of $F$. verticillioides were compared $24 \mathrm{~h}$ after inoculation, and the fungal growth rate in extracts from Trichoderma-inoculated plants showed a reduction of up to $50 \%$ as compared with that in extracts from control plants (Fig. 1). This reduction was paralleled by differential accumulation of anthocyanins and flavonoids (data not shown). After a careful evaluation of the results, we established maize inbred line $\mathrm{B} 73 / T$. atroviride/F. verticillioides as our model system.

\section{RNA-seq analysis and reads mapping to the maize genome.}

The ability of some Trichoderma spp. to activate local and systemic resistance processes in maize has been previously reported (Ferrigo et al. 2014). Here, transcriptional studies on 
T. atroviride-root-inoculated maize plants were conducted $24 \mathrm{~h}$ after $F$. verticillioides infection to further investigate the distant activation of plant immune responses. To better understand plant treatments, maize growth and the inoculation or infection protocol are summarized in Figure 2A. Four different conditions were analyzed by RNA-seq: control with no fungal treatment $(\mathrm{TmFm}), T$. atroviride inoculated $(\mathrm{TpFm}), F$. verticillioides infected (TmFp), and $F$. verticilliodes infected plus $T$. atroviride inoculated (TpFp) plants (Table 1). Overall, almost 350 million 100-bp reads were obtained, showing on average high sequencing quality (Phred scores $>30$ ). The alignment rate to the maize reference genome ranged from 67 to $79 \%$, being $73 \%$ on average. Except for sample TpFp3, all samples showed uniform values regarding mapping statistics. On average, $48 \%$ of the mapped reads were successfully assigned to known proteincoding transcripts as annotated in the Zea mays B73 reference genome (Supplementary Table S1). The correlation chart and dendrogram, based on global levels of expression, showed that the variability among samples was mainly explained by the infection with $F$. verticillioides (Supplementary Fig. S2).

\section{Maize differentially expressed genes.}

Three sets of significantly differentially expressed (DE) genes (adjusted $P$ value $<0.05$ and $\log _{2}$ fold change $[\mathrm{FC}]>1.5$ ) were identified: genes that responded to $F$. verticillioides infection (TmFp/TmFm), genes that responded to T. atroviride inoculation (TpFm/TmFm), and genes that responded to both $F$. verticillioides infection and $T$. atroviride inoculation (TpFp/TmFm). In total, 652 genes were identified as DE in at least one of the three contrasts: 332 were identified in TmFp/TmFm (191 upregulated and 141 downregulated), 559 in

Table 1. Inoculation and infection protocol of maize plants (inbred line B73) showing the four treatments used ${ }^{\mathrm{a}}$

\begin{tabular}{lcccc}
\hline & TpFp & TpFm & TmFp & TmFmb $^{\mathbf{b}}$ \\
\hline Trichoderma atroviride & + & + & - & - \\
Fusarium verticilliodes & + & - & + & - \\
\hline
\end{tabular}

${ }^{\mathrm{a}} \mathrm{T}=$ Trichoderma, $\mathrm{F}=$ Fusarium, $\mathrm{p}$ or + indicate with fungal treatment, and $\mathrm{m}$ or - indicate without fungal treatment.

b Control plants (without Trichoderma inoculation and without Fusarium infection)

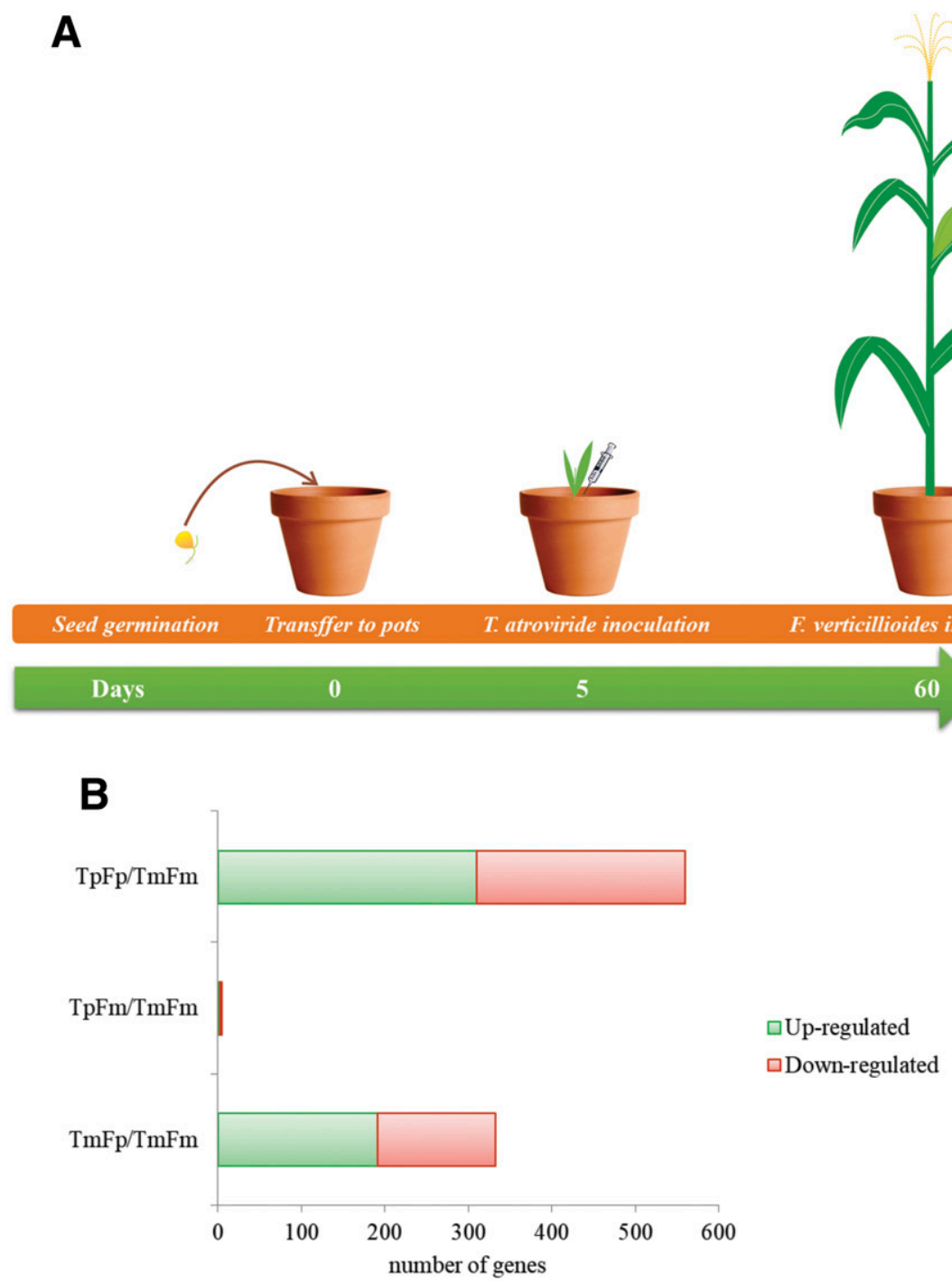

Fig. 2. Genes differentially expressed ( $\log _{2}$ fold change $>|1.5|$ and $P$-adj $\left.<0.05\right)$ in silks in response to Trichoderma atroviride or $F$. verticillioides. A, Cartoon depicting plant growth and inoculation or infection schedule. $\mathbf{B}$, Bar charts in which the number of upregulated and repressed genes is represented for three conditions: T. atroviride inoculated (TpFm), F. verticillioides infected (TmFp), and F. verticilliodes infected plus T. atroviride inoculated (TpFp), with respect to the control condition (no fungal treatment) (TmFm). C, Venn diagram summarizing the distribution of the 652 differentially expressed genes. 
TpFp/TmFm (310 upregulated and 249 downregulated), and 5 in $\mathrm{TpFm} / \mathrm{TmFm}$ (3 upregulated and 2 downregulated) (Fig. 2).

A global analysis using the Venn Diagram (Fig. 2C) indicated that only 2 out of all the DE genes (GRMZM2G125747 and GRMZM2G394203) were common to all the contrasts, and that 240 were shared between $\mathrm{TmFp} / \mathrm{TmFm}$ and $\mathrm{TpFp} / \mathrm{TmFm}$. A detailed analysis and conclusions for each situation are provided as Supplementary Text S1.

After transcriptional profiling, RNA-seq results were validated by quantitative reverse-transcription polymerase chain reaction (qRT-PCR), using a set of 10 genes identified as differentially regulated in the comparisons described earlier. Overall, the expression profiles determined by qRT-PCR displayed trends similar to those determined by RNA-seq for upregulated genes (Fig. 3). However, the difference in expression patterns for downregulated genes may be related to mRNA stability.

\section{Early transcriptional changes after pathogen challenge in maize silks under a primed state of defenses.}

The expression data of the DE genes were surveyed by cluster analysis, and genes were grouped into four clusters based on their expression behavior (Fig. 4). Cluster 1 grouped genes displaying similar $\log _{2} \mathrm{FC}$ values in $\mathrm{TmFp}$ and $\mathrm{TpFp}$ treatments compared with control plants (included in $\mathrm{TmFp} / \mathrm{TmFm}$ and $\mathrm{TpFp} / \mathrm{TmFm}$ lists, respectively) but not displaying DE in T. atroviride-only inoculated plants (those in the $\mathrm{TpFm} / \mathrm{TmFm}$ gene list), cluster 2 grouped genes displaying greater $\log _{2} \mathrm{FC}$ values in $\mathrm{TpFp} / \mathrm{TmFm}$ than in $\mathrm{TmFp} / \mathrm{TmFm}$, cluster 3 grouped genes exclusively detected as DE after $F$. verticilliodes infection ( $\mathrm{TmFp} / \mathrm{TmFm})$, and cluster 4 grouped genes exclusively detected as DE in TpFp plants (TpFp/TmFm gene list).

Based on this analysis, we focused on the 339 genes regulated by priming, which were those grouped in clusters 2 and 4 . Thus, this classification included all of the genes exclusively activated or deactivated in TpFp plants, as well as those with a significant DE when comparing the gene expression in TpFp plants versus that in TmFp plants (Mathys et al. 2012; Perazzolli et al. 2012; Pieterse et al. 2014). This behavior indicates an enhancement of maize immunological responses elicited by Trichoderma pretreatment. The whole set of genes identified as priming-related genes is detailed in Supplementary Table S2.

Transcriptomic analysis using MapMan 3.6.0 software (Thimm et al. 2004) allowed the functional classification of DE genes (Fig. 5). Accordingly, the most represented categories corresponded to cell structure or cell wall, amino acid and protein metabolism, response to stress, signaling, and transport $(8.5,7.5,6,9.5$, and $13 \%$, respectively). It is important to highlight that 10.5 and $23 \%$ of the total priming genes (339 genes) corresponded to miscellaneous or unknown functions, respectively.

\section{Specific gene groups differentially expressed in response to ISR.}

Primary metabolism. Relatively few genes involved in primary metabolism displayed significant differences (Fig. 6A). Downregulation of the following genes was evident when comparing the transcriptional profile of $\mathrm{TpFp} / \mathrm{TmFm}$ versus that of TmFp/TmFm: UTP-glucose-1-phosphate uridylyltransferase 1 (GRMZM2G098370), pyrophosphate:fructose6-phosphate 1-phosphotransferase (GRMZM2G042502), a dihydrolipoamide acetyltransferase coding for a component of pyruvate dehydrogenase complex (GRMZM2G058702), a plastid ADP glucose pyrophosphorylase large subunit
(GRMZM2G391936), and various starch synthesis-related genes. This response was likely due to reprogramming of cell carbon partitioning between storage and energy production to efficiently counteract pathogen invasion upon ISR activation.

Secondary metabolism. Transcriptional changes in secondary metabolism are of major interest because they are either directly or indirectly involved in the synthesis of antimicrobial compounds. In this category, the analysis was focused on the biosynthesis of waxes, terpenes, flavonoids, and phenylpropanoids. Several genes involved in the biosynthesis of waxes and terpenes such as glossyl (GRMZM2G114642) and terpene synthase (GRMZM2G010356) were upregulated by ISR. However, a gene coding for cinnamyl-alcohol dehydrogenase (GRMZM2G046070), an enzyme responsible for the final step in a branch of the phenylpropanoid pathway specific for the production of lignin monomers, was downregulated. In addition, the chalcone synthase profile was expected to be modified as shown by the downregulation of GRMZM2G151227. The evidence also demonstrated that a specific reprogramming of terpene and flavonoid metabolisms occurred in silk cells. It seems that, to produce a more effective battery of antimicrobial and signaling compounds, silks require a specific and tailored synthesis of secondary metabolites during ISR. It is likely that specific secondary metabolites are produced to counteract $F$. verticillioides in maize cells under an immunological alertness state.

Plant cell wall. During pathogen attack, the cell wall integrity is compromised and one of the responses is the reinforcement of the cell-wall structure. Genes coding for a bifunctional UDP-glucose 4-epimerase and UDP-xylose 4epimerase 1 (GRMZM2G052336), involved in the biosynthesis of colanic acid precursors, were upregulated under priming conditions. In contrast, many genes such as those coding for a putative mannan synthase (GRMZM2G099088), a putative cellulose synthase-like family protein (GRMZM2G015886), and a xyloglucan endotransglucosylase/hydrolase protein (ac210669.3_fgt001) were downregulated. We also identified two genes coding for $\beta$-D-xylosidase (GRMZM2G136895 and GRMZM2G111998) and three genes coding for expansins (GRMZM2G105844, GRMZM2G056236, and GRMZM2G082520), which were downregulated. These proteins are important for cell-wall loosening and recycling during plant growth. These observations would be in line with a decrease in the enzymes involved in the biosynthesis of structural polysaccharides and a general metabolic reorganization, likely to reinforce a physical barrier to prevent pathogenic invasion.

Defense genes. Most of the genes related to biotic stress detected as ISR-related, particularly several genes coding for PR proteins such as PR-1 (GRMZM2G304442) and PR-5 (GRMZM2G402631), thaumatin family proteins (GRMZM2G092474 and GRMZM2G136372), protein P21 (GRMZM2G039639), stress-induced protein 1 (GRMZM2G374971), chitinase 1 (GRMZM2G358153), endochitinase A (GRMZM2G051943), acidic endochitinase (GRMZM2G133781), win1 (GRMZM2G117989), xylanase inhibitor protein 1 (GRMZM2G328171), and glucan endo$\beta$-1,3-glucanase (GRMZM2G125032) were upregulated (Fig. $6 \mathrm{~B})$. This confirms that the primary defensive response of maize silks is to counteract pathogenic invasion by releasing an enzymatic arsenal against Fusarium spp. cell walls for two reasons: i) to attempt to prevent pathogen development and ii) to reinforce host defenses by the release of microbe-associated molecular patterns (MAMP) or pathogen-associated molecular patterns (PAMP) after pathogen cell-wall degradation.

Redox metabolism. It is known that redox metabolism plays an important role in the immune response in plants. Among the genes classified as ISR-responsive, two genes coding for 

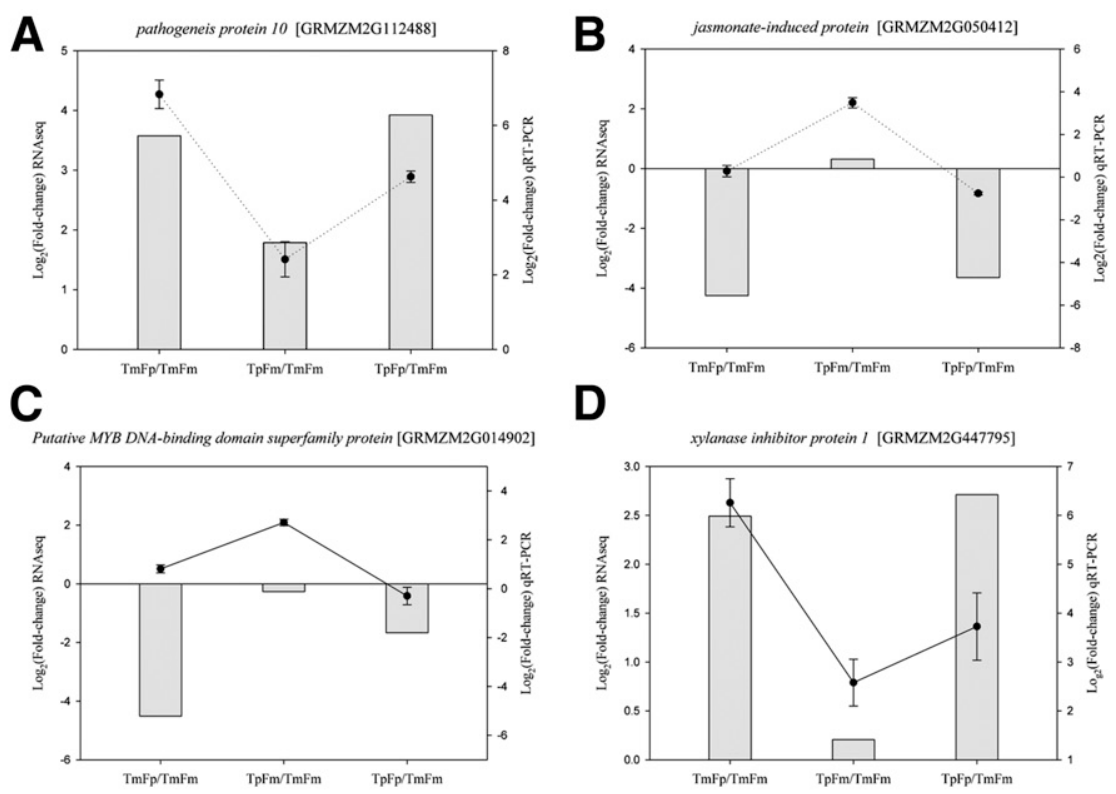

D
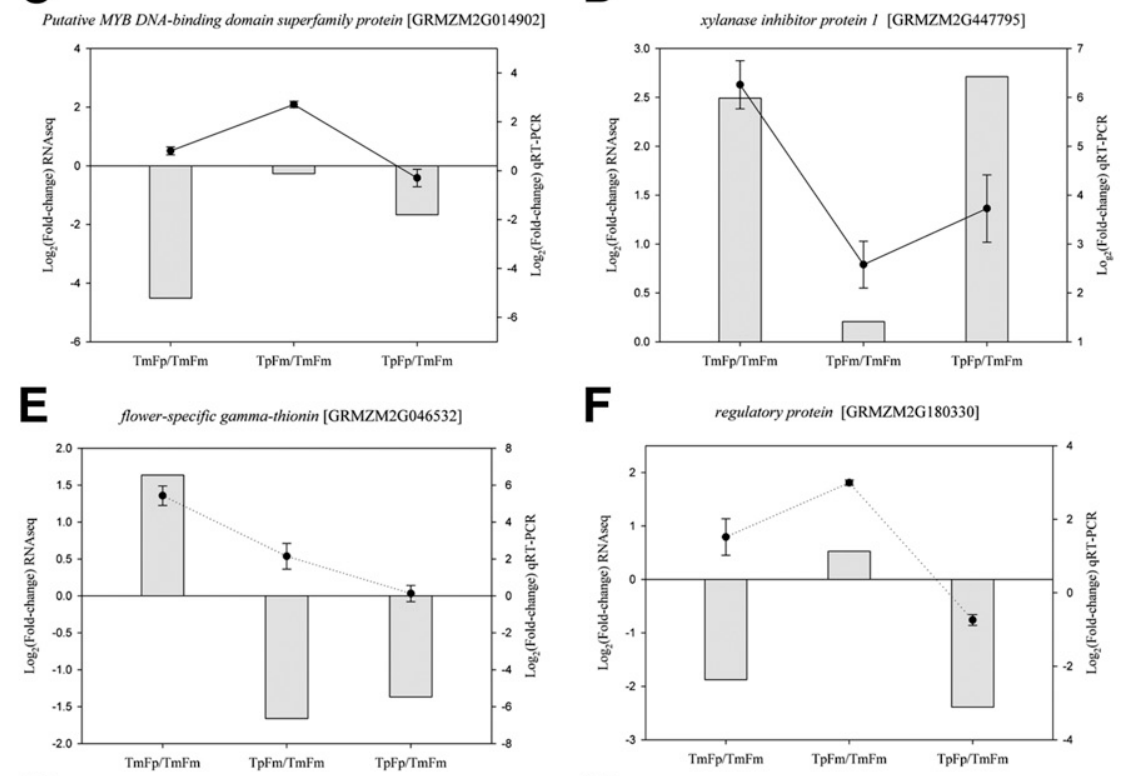

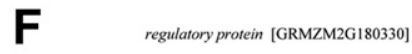

\section{G}

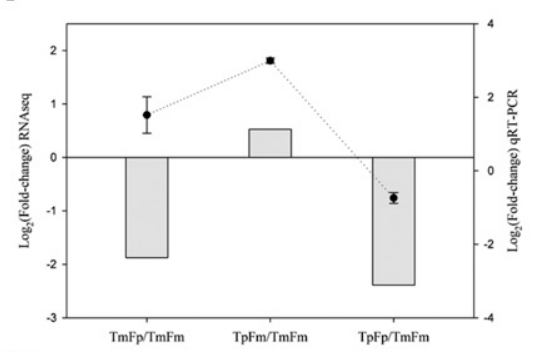

H
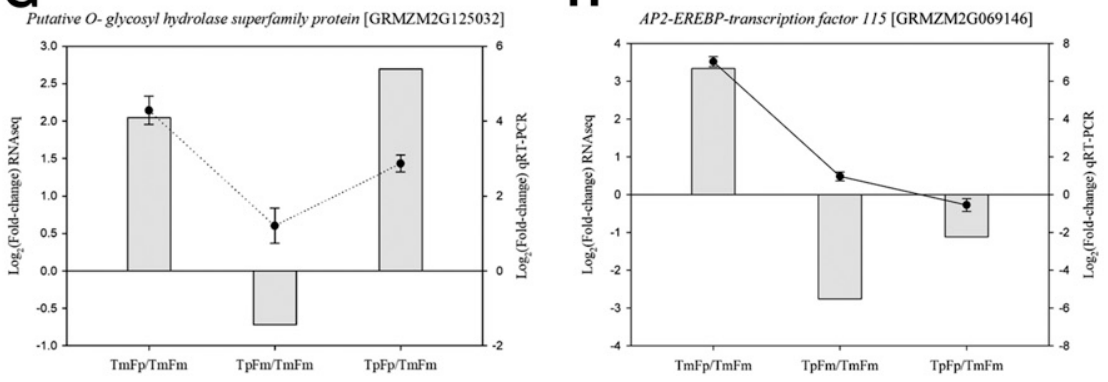

I
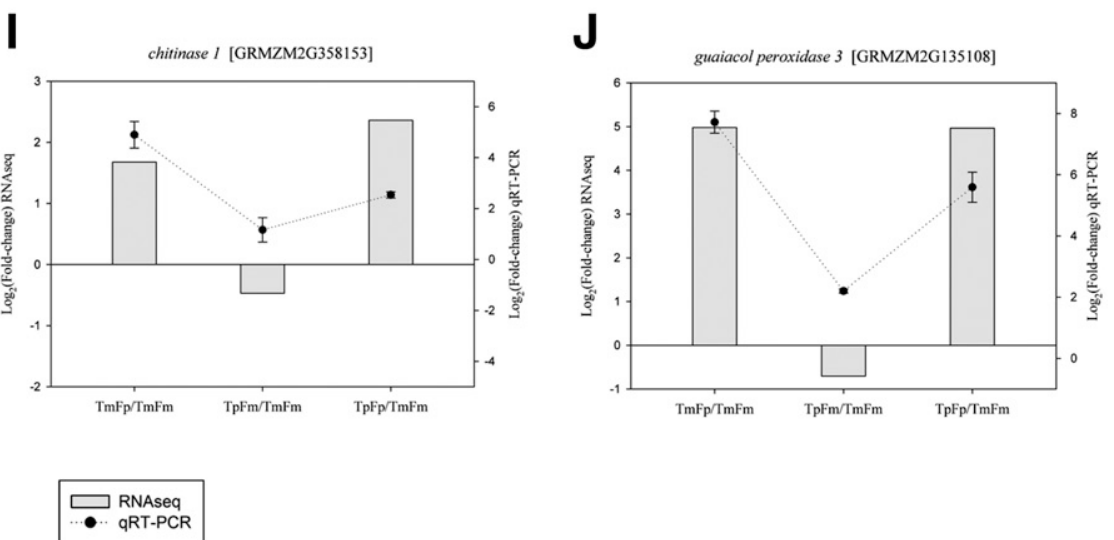

Fig. 3. Validation of RNA-seq results. A selected set of genes with differential expression patterns was assayed by quantitative reverse-transcription polymerase chain reaction (qRT-PCR) to confirm the transcriptional changes in maize silks due to Trichoderma atroviride or Fusarium verticillioides treatments. Selected genes code for A, pathogenesis protein 10 (GRMZM2G112488); B, jasmonate-induced protein (GRMZM2G050412); C, putative MYB DNA-binding domain superfamily protein (GRMZM2G014902); D, xylanase inhibitor protein 1 (GRMZM2G447795); E, flower-specific $\gamma$-thionin (GRMZM2G046532); F, regulatory protein (GRMZM2G180330); G, putative O-Glycosyl hydrolase superfamily protein (GRMZM2G125032); H, AP2-EREBP-transcription factor 115 (GRMZM2G069146); I, chitinase 1 (GRMZM2G358153); and J, guaiacol peroxidase 3 (GRMZM2G135108). Lines and histograms represent the relative expression levels ( $\log _{2}$ fold change) as assessed by qRT-PCR and RNAseq, respectively. The figure depicts the mean value \pm standard deviation of three independent replicates. 
peroxidase were modified: one was upregulated (ac205154.3 fgt005) whereas the other was downregulated (GRMZM2G023840). Also, the gene coding for APx3-peroxisomal ascorbate peroxidase (GRMZM2G004211) was downregulated. Three genes coding for glutathione transferase were observed: two of them (GRMZM2G119499 and GRMZM2G096269) were downregulated, whereas the other (GRMZM2G032856) was upregulated. Also, GRMZM2G135385, which codes for a cytochrome b5, was upregulated in response to priming in silk cells. These findings are in line with the role of glutathione and reactive oxygen species (ROS) at early stages of maize-fungus interaction (Vargas et al. 2012). Overall, these results demonstrate a strong reorganization of redox homeostasis in maize silks under a primed state in response to a pathogenic attack.

Transcription factors. An important outcome of this transcriptional analysis was the identification of several transcription factors differentially expressed as a consequence of ISR. These regulatory elements play critical roles in the setup of the

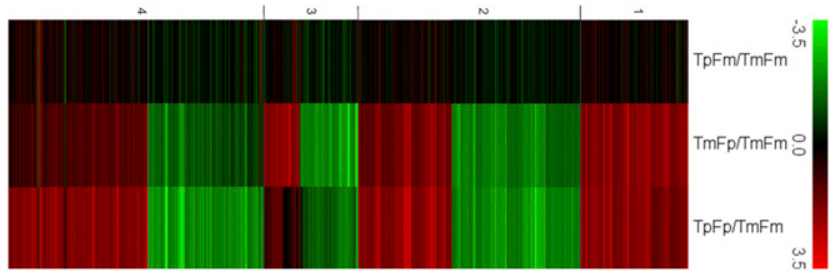

Fig. 4. Clustering of silk differentially expressed (DE) genes into four groups based on expression profiles. $\mathrm{TpFm}=$ Trichoderma atroviride in oculated, $\mathrm{TmFp}=$ Fusarium verticillioides infected, $\mathrm{TpFp}=$ F. verticilliodes infected plus T. atroviride inoculated, and $\mathrm{TmFm}=$ control $($ no fungal treatment). Cluster 1: Genes with similar values of DE both in $\mathrm{TmFp} / \mathrm{TmFm}$ and $\mathrm{TpFp} / \mathrm{TmFm}$ comparisons, and genes that do not show $\mathrm{DE}$ in the TpFm/TmFm comparison. Cluster 2: Genes that show DE in the $\mathrm{TpFp} / \mathrm{TmFm}$ comparison with values greater than those obtained in the $\mathrm{TmFp} / \mathrm{TmFm}$ comparison. Cluster 3: Genes with DE only for the F. verticillioides infection group $(\mathrm{TmFp} / \mathrm{TmFm})$. Cluster 4: Genes with $\mathrm{DE}$ only in the $\mathrm{TpFp} / \mathrm{TmFm}$ comparison. transcriptional reprogramming and the immune response enhancement after the pathogenic attack in primed cells. Several types of transcriptional factors, including Aux/indole 3-acetic acid (IAA), basic-domain leucine-zipper (bZIP), ET-responsive element-binding factors (ERF), MYB, and WRKY, have been reported to be involved in plant-pathogen interactions (Eulgem and Somssich 2007; Kazan and Manners 2009; Singh et al. 2002). However, ISR responses in maize silks also included specific changes in genetic elements involved in gene expression regulation. For instance, the expression of a gene coding for an ET-responding AP2-EREBP-transcription factor (GRMZM2G085678) was downregulated (Fig. 6B), whereas three genes coding for Aux/IAA, bZIP, and WRKY transcription factors (GRMZM2G001799, GRMZM2G073427, and GRMZM2G040298, respectively) were upregulated during the ISR activation.

Receptor kinases. RNA-Seq results and priming-related gene analysis allowed the detection of specific receptor kinases that likely act as cell-wall pathogen sensors such as LRR, DUF26, and cysteine-rich receptor (CRK) (Wrzaczek et al. 2010). These membrane-associated kinases play critical roles during signal perception and activation of the immune system. Specific brassinosteroid-related signaling events are upregulated during ISR responses, as shown by the induction of a coreceptor associated with LRR receptor-like kinases (BRASSINOSTEROID INSENSITIVE 1-associated receptor kinase 1, GRMZM2G015933, and BAK1), while many others were downregulated (GRMZM2G119850, GRMZM2G463904, GRMZM2G120657, GRMZM2G099981, GRMZM2G447447, GRMZM2G371137, GRMZM2G145753, and GRMZM2G138338). In parallel, four additional genes (GRMZM2G087625, GRMZM2G140231, GRMZM2G177726, and GRMZM2G340048) coding for the DUF26 protein family were upregulated in our selection of priming genes. In the case of GRMZM2G087625, its expression level in TpFp/TmFm doubled the $\log _{2} \mathrm{FC}$ value calculated in TmFp/TmFm. This observation indicates the importance of the gene product in signaling transduction at the early stages of the infection in silks under ISR.

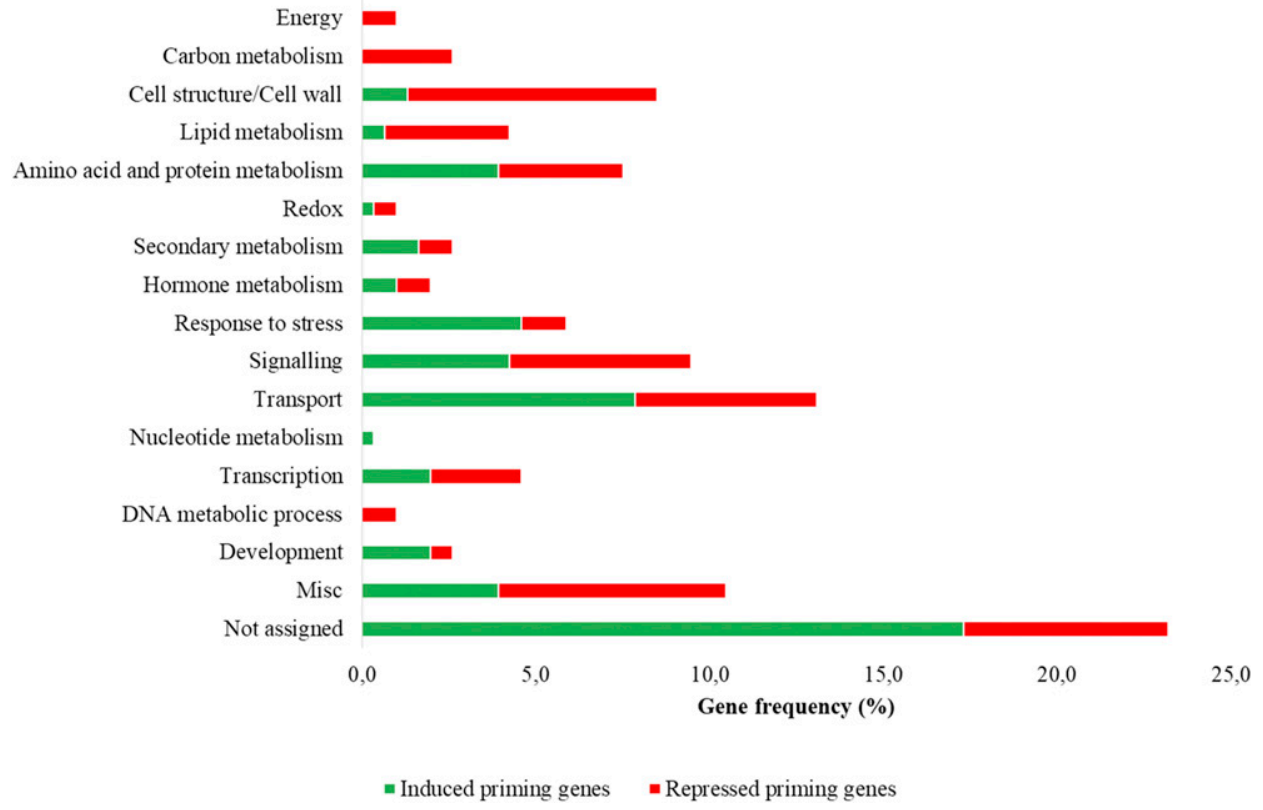

Fig. 5. Functional categories of those genes related to priming in maize silks. These categories were assigned based on MapMan results. The horizontal axis represents the percentage of induced or repressed genes in each functional category. 
After signal perception at the cell surface, secondary messengers (such as calcium) are critical for signal amplification and transduction (Lanubile et al. 2014). In this context, transcripts involved in the calcium-mediated signal transduction pathways were identified. Genes coding for an SF16 protein with the IQ calmodulin-binding motif (GRMZM2G024799), calreticulin (GRMZM2G074687), and putative calciumdependent protein kinase family protein (GRMZM2G030673) were detected as differentially downregulated when TpFp/TmFm was compared with $\mathrm{TmFp} / \mathrm{TmFm}$. This phenomenon seems to
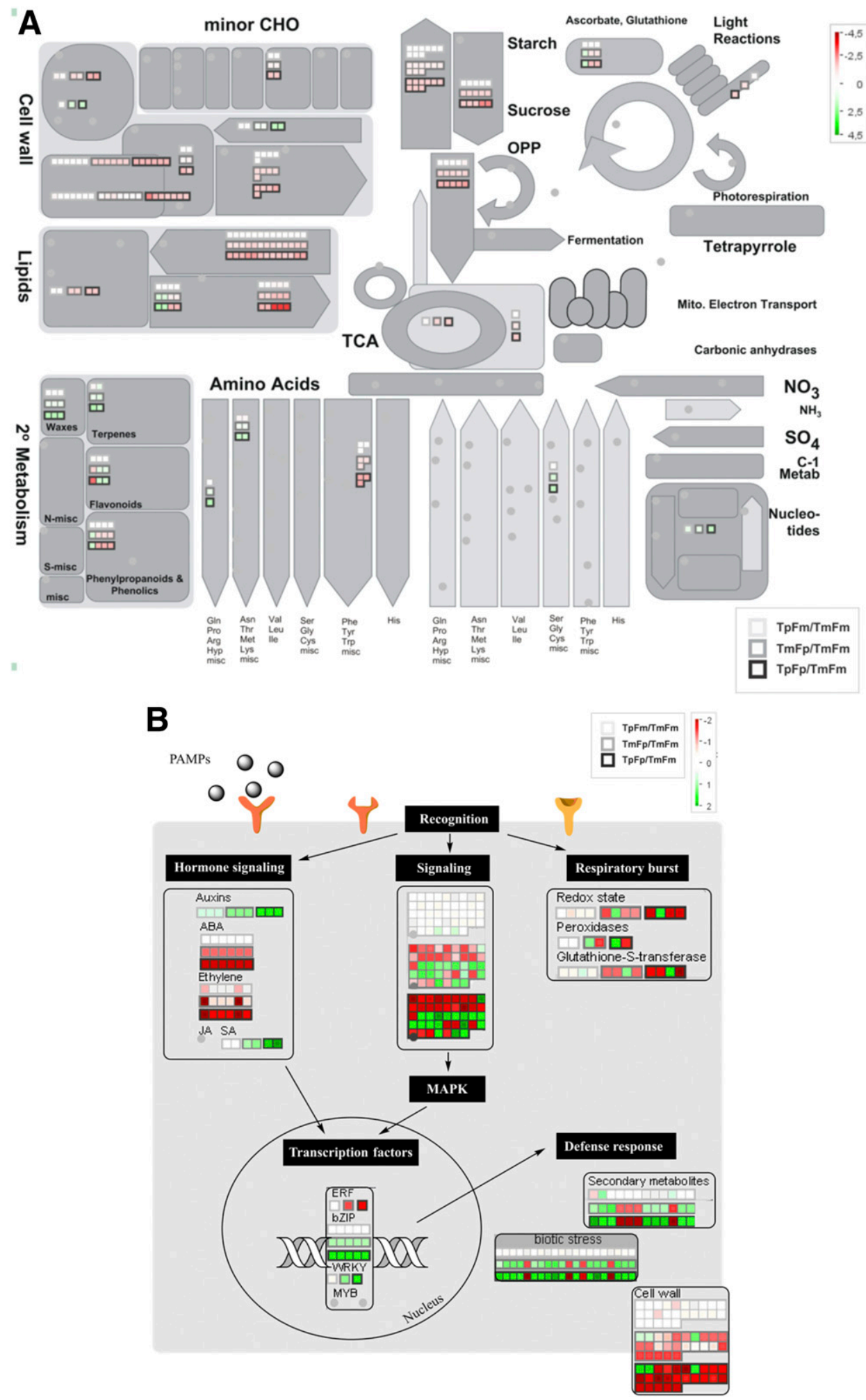

Fig. 6. Overview of transcriptional changes in maize silks. A, General metabolic changes. $\mathrm{TpFm}=$ Trichoderma atroviride inoculated, TmFp $=$ Fusarium verticillioides infected, $\mathrm{TpFp}=F$. verticilliodes infected plus $T$. atroviride inoculated, and $\mathrm{TmFm}=$ control (no fungal treatment). B, Biotic stress-related transcriptional modifications. Transcriptional changes are observed in a shaded scale. Light and dark boxes represent upregulated and downregulated genes, respectively. Every treatment is depicted with various frame shading as follows: white for $\mathrm{TpFm} / \mathrm{TmFm}$, light gray for $\mathrm{TmFp} / \mathrm{TmFm}$, and black for $\mathrm{TpFp} / \mathrm{TmFm}$. 
indicate that some $\mathrm{Ca}^{2+}$-induced pathways are attenuated in maize silks after ISR activation.

Hormone signaling. Based on MapMan analysis, several genes related to abscisic acid (ABA) and ET signaling were downregulated when ISR was activated (Fig. 6B). Particularly, a gene coding for a protein in the ABA-responsive HVA22 family (GRMZM2G101062) and two genes (GRMZM2G007249 and GRMZM2G013448) coding for the enzyme 1-aminocyclopropane-1-carboxylate oxidase, responsible for ET biosynthesis, displayed lower expression levels in the TpFp/TmFm condition. In contrast, SA and IAA signaling events appeared to be enhanced in primed silks after pathogen attack. In the evaluation of auxin-related pathways, IAA biosynthesis seemed to be activated, because the gene coding for IAA amino acid hydrolase ILR1 (GRMZM2G016958) was upregulated. Similarly, two genes coding for salicylate/benzoate carboxyl methyltransferase (BSMT) (GRMZM2G010731 and GRMZM2G126772), responsible for SA methyl-ester (MeSA) formation, were upregulated. MeSA has been proposed to act as a volatile systemic messenger that is produced at the infection site by BSMT1 and transduced to systemic tissues, where it is converted back to SA to activate defenses in noninfected parts of the plant (Gao et al. 2014; Park et al. 2007).

Finally, no genes involved in the JA signaling pathways were found among the priming-related genes in silk cells $24 \mathrm{~h}$ postinfection. It is likely that, in this tripartite system, the responses mediated by JA are not fully developed at early stages of the pathogenic attack and a more detailed analysis will be required.

\section{Analysis of SA, JA, and ABA as the main players in ISR-mediated immunity in maize silks.}

To gain insights into the hormonal changes occurring after each treatment, we compared the levels of SA, JA and ABA in the silks of the four treatments studied (Fig. 7). The most significant changes were observed in the SA levels (Fig. 7A). It is worth mentioning that, at early stages of the infection process, $F$. verticillioides acts as a biotrophic pathogen leading to the activation of the SA pathway (Glazebrook 2005). This is concordant with the significant increase in the levels of this hormone detected in the TmFp treatment compared with the control, TmFm. TpFp presented lower SA levels than TmFp and TpFm and similar to the control condition $(\mathrm{TmFm})$. This reduction in SA content agrees with the increased levels of the BSMT gene, whose protein product transforms SA into MeSA, a signal molecule for longdistance communication.
Regarding JA levels, it seems that the main effect was that exerted by $F$. verticillioides infection (Fig. 7B). In agreement with this, no significant differences in JA levels were detected between TpFm and TmFm, while similar values were measured for TmFp and TpFp. Therefore, Fusarium infection produces similar JA levels independently of the Trichoderma treatment, which is in agreement with previous reports that no major changes in JA were detected in plants expressing ISR (Pieterse et al. 2000). On the other hand, the analysis of ABA levels showed no significant changes $(P$ value $<0.05)$ between TmFp, TpFm, and TpFp with respect to the control, TmFm (Fig. 7C). Based on both hormone and transcriptional analyses, it seems that, for the maize/Trichoderma/Fusarium tripartite system, the priming responses in maize silks may be related to the SA and, to some extent, the JA signaling pathways.

To further support this concept, the transcriptional behavior of key genes involved in the hormonal synthesis and signaling pathways of both SA and JA was further investigated. By relaxing the criteria to consider significant DE genes those with $p$-adj $<0.05$ but regardless the $\log _{2} \mathrm{FC}$, we found 33 and 22 additional genes related to the $\mathrm{SA}$ and JA pathways in $\mathrm{TpFp} / \mathrm{TmFm}$ and $\mathrm{TmFp} / \mathrm{TmFm}$, respectively (data not shown).

The signaling pathway of SA was in general upregulated during ISR in maize silks (Supplementary Fig. S3). For instance, the gene coding for the master regulator NPR1/NH1 (GRMZM2G077197), which interacts with TGA transcription factors (and activates plant defenses), was slightly increased in the $\mathrm{TpFp} / \mathrm{TmFm}$ condition. In addition, two genes coding for transcriptional factors homologous to WRKY13 (GRMZM2G013391 and GRMZM2G141299) were upregulated in the $\mathrm{TpFp} / \mathrm{TmFm}$ condition. In Oryza sativa (Cheng et al. 2015), WRKY13 works as a transcriptional repressor of WRKY45/WRKY45-1. Then, in good agreement with WRKY13 upregulation, in our tripartite system, WRK45/WRKY45-1 was downregulated in the same condition. Regarding the synthesis of SA, it has been reported that NPR1 negatively regulates the expression pathway of isochorismate synthase 1 (a crucial enzyme for SA biosynthesis) by a mechanism that is still unknown (Wildermuth et al. 2001; Zhang et al. 2010). Then, the negative effect on SA biosynthesis together with higher levels of BSMT would be in good agreement with the lower levels of SA detected in TpFp (Fig. 7A). It is likely that, during ISR responses, SA levels reach a threshold level determined by the balance between the biosynthetic rate and its transformation into MeSA (Park et al. 2007). In this context, SA homeostasis would play a central role in the local and distant signaling upon $F$. verticillioides infection in ISR-expressing silks.
A

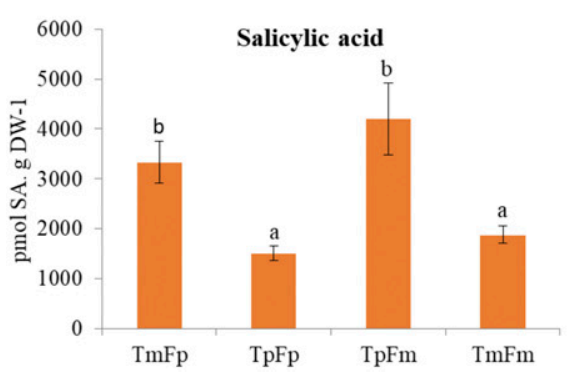

B

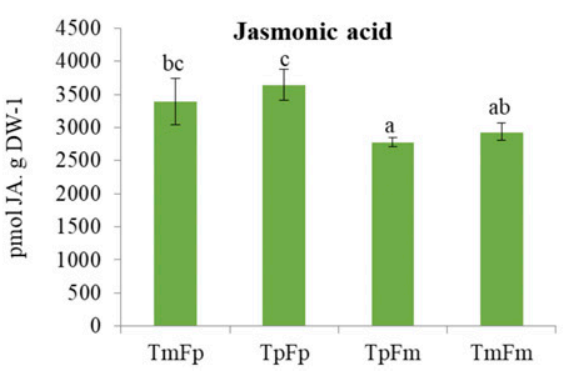

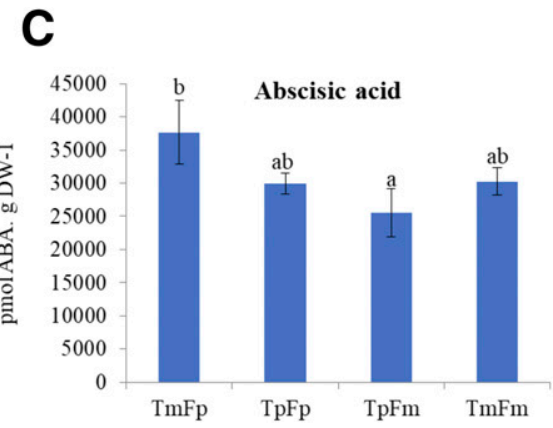

Fig. 7. Hormone levels in maize silks after fungal treatment. TpFm $=$ Trichoderma atroviride inoculated, TmFp $=$ Fusarium verticillioides infected, TpFp $=$ F. verticilliodes infected plus T. atroviride inoculated, and TmFm = control (no fungal treatment). A, Salicylic acid (SA); B, jasmonic acid (JA); and C, abscisic acid (ABA). Hormone levels are reported as picomoles per gram dried weight (DW) of silks. Bars depict the mean value \pm standard deviation of three independent replicates. The hormone quantification was performed by high-performance liquid chromatography mass spectrometry according to protocols described in Material and Methods. Bars with different letters, differ significantly at a significance level of 5\% according to Tukey's test. 
After a JA signaling pathway survey, an increase in the expression of the genes coding for the repressor JAZ, the corepressor TPL, and the adaptor protein NINJA was evident in the $\mathrm{TpFp} / \mathrm{TmFm}$ condition. However, in most cases, the increase observed in $\mathrm{TpFp} / \mathrm{TmFm}$ was greater than in the $\mathrm{TmFp} / \mathrm{TmFm}$ condition, which could be attributed to the effect of priming. These genes are known to be critical repressors of expression modulated by the MYC (wounding-response genes) and ERF (pathogen-response genes) transcription factors. In our case, the upregulated levels of the MYC transcription factor would be inhibiting the expression of the genes induced by the ERF transcription factor. Accordingly, the gene encoded by GRMZM2G046532 (with 65.7\% identity to PDF2.1) was downregulated in maize silks under ISR.

\section{DISCUSSION}

ISR is one of the most intriguing processes activated in plants when interacting with beneficial microorganisms. This phenomenon has been investigated for many years but the regulatory and metabolic aspects are not yet fully understood (Conrath 2011; Mathys et al. 2012; Nawrocka and Małolepsza 2013; Pieterse et al. 2014). To gain new insights into the molecular mechanisms involved in ISR responses in plants, here we defined a model system based on the worldwide importance of maize and the strong impact of $F$. verticillioides on crop yield and food safety (Lanubile et al. 2017).

It is widely known that no maize line is fully resistant to Fusarium sp. attack; however, many studies have demonstrated the beneficial effects of some Trichoderma spp. as biological agents to reduce Fusarium infection (Ferrigo et al. 2014; Saravanakumar et al. 2016). In this context, the effect of Trichoderma spp. may be attributed to either a direct antagonism of Fusarium spp. on soil inocula or the induction of a heightened state of plant immunity (Djonović et al. 2007; Ferrigo et al. 2014). However, the mechanisms underlying the effects of Trichoderma spp. on maize are not yet fully understood. Thus, the focus of this research was to globally explore the early regulatory events modulated by ISR in maize silks after $F$. verticillioides infection, through a transcriptional approach.

Our findings allowed the identification of specific responses regulated by ISR in plants primed at the seedling stage and pathogen-challenged 2 months later (during the transition from the vegetative to the reproductive stage, VT to R0-R1). Functional categories of DE genes included transport and signaling, amino acid and protein metabolism, and biotic or abiotic stress response (Fig. 5). This clearly demonstrates a mandatory and urgent metabolic reorganization of plant cells to mount a quicker and more efficient immunological response.

In good agreement with the hallmark of ISR, where the primed state in unchallenged plants is often imperceptible (Pieterse et al. 2014), only five genes displayed differential mRNA levels (Fig. 2C) in silks of T. atroviride-inoculated plants. The identification of these genes highlighted the importance of cell wall metabolism and steroid-mediated signaling events in the alertness state of silks during ISR. The activation of a gene coding for a cell wall structural protein is in agreement with an enhanced plant cell-wall metabolism and structural reinforcement to reduce pathogen ingress, one of the widely known effects of Trichoderma spp. after plant root colonization (Harman et al. 2004). The activation of a gene coding for a putative cycloartenol synthase, which converts (S)2,3-epoxysqualene into cycloartenol, also unraveled the importance of steroid-based secondary metabolism in the heightened state of defense in plants. Cycloartenol is an important triterpenoid of the sterol class found in plants and is the starting point for the synthesis of most plant steroids (Gas-
Pascual et al. 2014; Schaller 2003). The distant and long-lasting enhancement of steroid metabolism mediated by Trichoderma spp. in maize is in agreement with previous reports on terpenes (and their derivatives) acting as signals related to attacks by pathogens or herbivores (Chen et al. 2011; Raguso 2009). The systemic effect of Trichoderma spp. on the secondary metabolism of plants has been mainly related to the accumulation of antimicrobial compounds (Druzhinina et al. 2011) but the role of steroid derivatives is likely to have a more specific role in the signaling and maintenance of the ISR status in distant tissues.

On the other hand, genes specifically regulated by ISR after pathogen challenge were evident when comparing genes listed in the $\mathrm{TpFp} / \mathrm{TmFm}$ versus the $\mathrm{TmFp} / \mathrm{TmFm}$ categories. The analysis of this gene core set allowed the discovery of a regulatory network that might be responsible for the activation and amplification of pathogen-related signals in plants under a heightened state of defense. This regulatory network involves the brassinosteroid coreceptor BAK1 (specific for brassinosteroid and MAMP signaling), cysteine-rich receptors from the DUF26 family, and several transcription factors.

BAK1 is a coreceptor acting together with BIR and other LRR receptors, which mediates responses elicited by brassinosteroids or by pathogen-released PAMP, such as Flg22 (Huot et al. 2014). Brassinosteroids have been defined as the sixth plant hormones, with analogous structure to animal steroid hormones, which play central roles in diverse aspects of plant biology, including immunity (He et al. 2007; Huot et al. 2014; Tang et al. 2016). Previous reports have demonstrated that, in Arabidopsis, brassinosteroid signaling cascades are involved in disease resistance against Pseudomonas syringae and the oomycete Hyaloperonospora arabidopsidis, in a PAMPtriggered immunity (PTI)-dependent mechanism (Roux et al. 2011). This observation suggests that, in maize silks expressing ISR, signaling events mediated by BAK1 might be key players in the recognition of endogenous or pathogen-derived signal molecules. Then, supporting the concept of the interplay between PTI and receptor kinases to elicit immunological responses in primed plants, several DUF26 homologs were also differentially regulated after pathogen challenge in maize plants under ISR. In many cases, receptors belonging to the DUF26 family have been related to the activation of PTI mechanisms. For instance, in Arabidopsis plants overexpressing the LecRKVI.2-responsive CRK4, CRK6, and CRK36, PTI mechanisms were enhanced and transgenic plants developed resistance to the pathogen $P$. syringae (Yeh et al. 2015).

According to our gene expression analysis, a synergistic effect between brassinosteroids and PAMP seemed to be occurring in maize plants to quickly intensify the activation of local immune responses during ISR. This conclusion is supported by the enhancement of the steroid biosynthetic pathway, the increased expression of the coreceptor BAK1, and the higher expression level of other PTI-related receptors after pathogen challenge.

Correlating with signal perception and transduction into the host cell, the expected outcome is a global physiological reorganization to cope with the stressful situation. In this context, transcriptional regulators were also detected as part of the responses mediated by ISR. Initial PTI responses are triggered within a few hours after PAMP perception and include an ROS burst, PR proteins, and other general immune responses (Huot et al. 2014). PTI-related transcriptional reprogramming has been partly associated with the WRKY family of transcription factors (Huot et al. 2014; Pandey and Somssich 2009). However, ISR-modulated genes include a WRKY protein together with a bZIP and an Aux/IAA regulator as the main transcription factors specifically upregulated after $F$. verticillioides infection of primed silks. 
Collectively, all of these results point to an intricate regulatory network activated in primed cells that locally works to prevent pathogen efforts to enter the plant cell. The perception of the pathogen hypha in the host cell surroundings seems to trigger three immediate molecular responses in an attempt to arrest pathogenic infection. First, antimicrobial compounds (such as ROS, flavonoids, and other secondary metabolites) are synthesized by maize cells to inhibit Fusarium growth inside maize cells. At the same time, maize cells reorganize their main physical barrier (the cell wall) to delay further pathogenic invasion. Finally, an arsenal of extracellular enzymes produced to degrade Fusarium spp. cell walls would assist to locally restrain pathogen growth and promote the generation of massive amounts of PTI elicitors.

Our transcriptome analysis together with the hormone quantitative determinations performed also shed some light on the role of hormones in the regulation of immune responses in maize silks after ISR activation. The most outstanding finding was an apparent combination of effects mediated by SA and JA signaling pathways. Contrary to previous studies performed in maize or other monocots (Contreras-Cornejo et al. 2011; Djonović et al. 2007; Hermosa et al. 2012; Salas-Marina et al. 2011; Pieterse et al. 2014), no evidence of the activation of specific ET-related pathways was detected in infected silks under ISR, and even the gene coding for the ET-biosynthetic enzyme was downregulated. On the other hand, based on hormone levels, it appears that SA plays a crucial role in longdistance signaling after pathogen infection. The evidence presented shows that $\mathrm{SA}$ is accumulated in silks of $T$. atrovirideinoculated plants or after F. verticillioides infection (Fig. 7A). However, during pathogen infection in primed plants ( $\mathrm{TpFp}$ ), the significant decrease in SA levels may be attributed to a downregulation of the biosynthetic pathway, together with the conversion of free SA into MeSA. The higher levels of the transcript for BSMT highlights that the lower levels of SA are associated with an increased synthesis of MeSA as a signal for noninfected tissues (Kumar 2014; Mauch-Mani et al. 2017; Park et al. 2007; Pieterse et al. 2014). The regulatory pathways under the influence of SA displayed three main changes specific to ISR and correlated to a decrease in SA levels: the transcriptional activation of the master regulator NPR1, the activation of PR1 (an SA marker gene), and the repression of WRKY76. On the other hand, JA-mediated effects on a primed immune system were connected to the transcriptional activation of JAZ, NINJA, and EIN3/EIL1 and the repression of a PDF2.1-like gene (GRMZM2G046532. These main transcriptional changes due to ISR also affected genes coding for additional accessory proteins, including COI1, MYC-like, and TPL, necessary to execute a global gene expression program to trigger defensive responses.

It is likely that, in maize silks, SA acts as a central signaling element to alert distant tissues during ISR responses. According to the data presented here, maize silks under a primed state of defense establish a strong battle to immediately confine $F$. verticillioides and, at the same time, release the volatile MeSA to trigger immune responses in noninfected tissues or neighbor plants.

Taken together, transcriptomic and hormonal studies highlighted steroids (likely brassinosteroids) and SA signaling pathways as the leading actors in maize silks to mediate local and systemic activation of defenses.

\section{MATERIALS AND METHODS}

\section{Fungal strains.}

Four reference strains of Trichoderma (T. virens Gv29-8, T. atroviride IMI206040, T. harzianum, and T. reesei Q6) and the $F$. verticillioides $\mathrm{P} 364$ isolate were grown on PDA (Difco) for 10 days at $25^{\circ} \mathrm{C}$. Conidia were harvested by gentle scraping in $2 \mathrm{ml}$ of sterile water, counted in a Neubauer chamber, and diluted in sterile water, as described elsewhere (Iglesias et al. 2010).

\section{Plant material.}

Maize (Z. mays, inbred line B73) seed were surface disinfected with $100 \%$ ethanol for $5 \mathrm{~min}$, followed by $4 \% \mathrm{NaClO}$ for $15 \mathrm{~min}$, washed five times with sterile distilled water, and germinated in a humidity chamber at $25^{\circ} \mathrm{C}$ in the dark. Healthy seedlings were transferred to soil and inoculated with the Trichoderma strains required for each case.

\section{Fungal inoculation and infection}

of maize plants for transcriptional analysis.

Germinated maize seed, with no evidence of fungal growth, were transferred to 30-liter pots containing sterile soil and, 5 days later, inoculated with $2 \mathrm{ml}$ of a T. atroviride spore suspension $\left(1 \times 10^{5}\right.$ spores $\left./ \mathrm{ml}\right)$. The pots were transferred to the greenhouse until the emergence of silks.

For pathogen inoculation, $2 \mathrm{ml}$ of a conidial suspension of $F$. verticillioides $\left(1 \times 10^{6}\right.$ conidia/ml $)$ was injected into the silk channel approximately 4 to 6 days after silking, by using the technology developed by Iglesias et al. (2010). The silks were collected $24 \mathrm{~h}$ postinfection and stored at $-80^{\circ} \mathrm{C}$. The inoculation and infection protocols are summarized in Table 1. The inoculation protocol was performed in three biological replicates consisting of five different plants for each condition. Each sample was prepared by pooling the silks of five different ears.

\section{RNA purification, library preparation, and RNA sequencing.}

Total RNA was extracted from $100 \mathrm{mg}$ of tissue using the Trizol protocol (Invitrogen) according to the manufacturer's instructions. The amount and quality of total RNA were estimated spectrophotometrically at 260 and $280 \mathrm{~nm}$ as well as by $1 \%$ agarose (wt/vol) gel electrophoresis. For quality control of RNA samples, $1 \mu \mathrm{l}$ of each sample was run on a 2100 Bioanalyzer (Agilent Technologies) using the Agilent RNA 6000 Peak Kit.

The TruSeq RNA Sample Preparation Guide from Illumina was used for RNA processing and library construction. According to the quality controls on the 2100 Bioanalyze and using a DNA $1000 \mathrm{Kit}$, the libraries were composed of fragments of double-stranded DNA of an average size of $260 \mathrm{bp}$. The libraries were quantified by qPCR (Light Cycler 480 Roche), using the Kapa Library Quantification Kit according to the manufacturer's instructions. An equimolar pool of the libraries was prepared and used for the generation of clusters in the sequencing cell.

RNA-seq analysis was performed by a fast sequencing run (two lanes) generating $2 \times 100$-bp paired-end reads in Hiseq 1500-Illumina.

\section{Bioinformatic analysis.}

RNA-seq raw sequence reads were quality-checked using FastQC (Andrews 2010). Fastq sequences showed an overall good quality according to the FastQC standards (mean Phred score $=37$ ). Raw fastq sequences were trimmed to remove both low-quality bases and adapter sequences at the $3^{\prime}$ end of the reads. Trimming was performed using Trimmomatic v.0.3 (Bolger et al. 2014), with the options leading:11, trailing:11, slidingwindow:4:20, and minlen:24. Tophat v2.0.8 (Kim et al. 2013) was used to map trimmed reads to the reference genome. The Ensembl release 27 of the Z. mays B73 (AGPv.3.27) was used as a reference genome and the corresponding annotation file (Kersey et al. 2016). FeatureCounts v.1.5 (Liao et al. 2014) was used to count reads at the gene level. Multimapping and 
ambiguously mapped reads were not considered. A differential gene expression analysis was performed using the DeSeq2 package (Love et al. 2014). The FC were calculated as the ratio of the normalized raw counts between each treatment and the control condition treatment ( $\mathrm{TmFm}$ ). Thus, we identified genes that responded to $F$. verticillioides infection (TmFp/TmFm), to T. atroviride inoculation ( $\mathrm{TpFm} / \mathrm{TmFm})$, or simultaneously to both $F$. verticillioides and $T$. atroviride $(\mathrm{TpFp} / \mathrm{TmFm})$. Genes with adjusted $P$ values $<0.05$ and $\log _{2}(\mathrm{FC}) \geq|1.5|$ were considered as significantly differentially expressed. These DE genes were then grouped according to their expression by comparing the behavior between these three conditions using the $k$-means clustering method of the T-MeV 4.8.1 software (Saeed et al. 2006).

This cluster analysis allowed detection of priming genes induced by Trichoderma spp. in silks of maize plants. These priming genes were visualized and functionally analyzed using MapMan 3.6.0 (Thimm et al. 2004).

\section{Hormone determination: ABA, JA, and SA.}

Hormones were extracted from lyophilized maize silks according to the protocol by Durgbanshi et al. (2005). An extraction solution containing $50 \mathrm{ng}$ of internal standards $\left[{ }^{2} \mathrm{H}_{6}\right]-$ ABA, $\left[{ }^{2} \mathrm{H}_{6}\right]-\mathrm{JA}$, and $\left[{ }^{2} \mathrm{H}_{4}\right]-\mathrm{SA}$ was used for sample preparation and high-performance liquid chromatography (HPLC) electrospray ionization tandem mass spectrometry was used for hormone identification.

The chromatography separation was performed with an HPLC Alliance 2695 (Waters, Inc.) equipped with a C18 reversephase column ( 100 by $2.1 \mathrm{~mm}, 3 \mu \mathrm{m}$ ), which is coupled with an electrospray ionization source, and a Triple Quadrupole Mass Spectrometer (Quattro Ultima pt; Micromass). Data obtained were in negative mode.

Identification and quantification were accomplished by injection of the samples in multiple reaction monitoring (MRM) mode. Data acquisition by MRM mode was carried out by monitoring the parental ions, and their transitions were: ABA: $263>153,\left[{ }^{2} \mathrm{H}_{6}\right]-\mathrm{ABA}: 268>159$, JA: $209>59,\left[{ }^{2} \mathrm{H}_{6}\right]-\mathrm{JA}: 215>$ 59, SA: $137>93$, and $\left[{ }^{2} \mathrm{H}_{4}\right]-\mathrm{SA} 141>97$.

Data were processed with MassLynx (v. 4.1; Micromass) and the values were determined as picomoles per gram of dried weight.

\section{ACKNOWLEDGMENTS}

We thank the staff of the English Department (Facultad de Ciencias Bioquímicas y Farmacéuticas-UNR) for the language correction of the manuscript; D. Presello from EAA-INTA Pergamino, Argentina for providing F. verticilloides $\mathrm{P} 364$; and C. M. Kenerley at Texas A\&M University for kindly providing Trichoderma strains. W. A. Vargas, V. A. CamposBermudez, and S. P. Rius are members of CIC from CONICET, Argentina; R. B. Agostini is a doctoral fellow from ANPCYT; and A. Postigo is a postdoctoral fellow from CONICET.

\section{LITERATURE CITED}

Andrews, S. 2010. FastQC: A quality control tool for high throughput sequence data. Online publication. https://www.bioinformatics.babraham.ac.uk/projects/fastqc/

Battilani, P., Pietri, A., Barbano, C., Scandolara, A., Bertuzzi, T., and Marocco, A. 2008. Logistic regression modeling of cropping systems to predict fumonisin contamination in maize. J. Agric. Food Chem. 56: 10433-10438.

Bolger, A. M., Lohse, M., and Usadel, B. 2014. Trimmomatic: A flexible trimmer for Illumina sequence data. Bioinformatics 30:2114-2120.

Bottalico, A. 1998. Fusarium diseases of cereals: Species complex and related mycotoxin profiles. Eur. J. Plant Pathol. 80:85-103.

Chen, F., Tholl, D., Bohlmann, J., and Pichersky, E. 2011. The family of terpene synthases in plants: A mid-size family of genes for specialized metabolism that is highly diversified throughout the kingdom. Plant J. $66: 212-229$.
Cheng, H., Li, H., Deng, Y., Xiao, J., Li, X., and Wang, S. 2015. The WRKY45-2 WRKY13 WRKY42 transcriptional regulatory cascade is required for rice resistance to fungal pathogen. Plant Physiol. 167: 1087-1099.

Chulze, S. N., Ramirez, M. L., Farnochi, M. C., Pascale, M., Visconti, A., and March, G. 1996. Fusarium and fumonisin occurrence in Argentinian corn at different ear maturity stages. J. Agric. Food Chem. 44:2797-2801.

Clements, M. J., Maragos, C. M., Pataky, J. K., and White, D. G. 2004 Sources of resistance to fumonisin accumulation in grain and fusarium ear and kernel rot of corn. Phytopathology 94:251-260.

Conrath, U. 2011. Molecular aspects of defence priming. Trends Plant Sci. $16: 524-531$.

Contreras-Cornejo, H. A., Macías-Rodríguez, L., Beltrán-Peña, E., HerreraEstrella, A., and López-Bucio, J. 2011. Trichoderma-induced plant immunity likely involves both hormonal- and camalexin-dependent mechanisms in Arabidopsis thaliana and confers resistance against necrotrophic fungi Botrytis cinerea. Plant Signal. Behav. 6:1554-1563.

de Medeiros, H. A., de Araújo Filho, J. V., de Freitas, L. G., Castillo, P., Rubio, M. B., Hermosa, R., and Monte, E. 2017. Tomato progeny inherit resistance to the nematode Meloidogyne javanica linked to plant growth induced by the biocontrol fungus Trichoderma atroviride. Sci. Rep. 7: Article 40216.

Djonović, S., Vargas, W. A., Kolomiets, M. V., Horndeski, M., Wiest, A., and Kenerley, C. M. 2007. A proteinaceous elicitor Sm1 from the beneficial fungus Trichoderma virens is required for induced systemic resistance in maize. Plant Physiol. 145:875-889.

Druzhinina, I. S., Seidl-Seiboth, V., Herrera-Estrella, A., Horwitz, B. A., Kenerley, C. M., Monte, E., Mukherjee, P. K., Zeilinger, S., Grigoriev, I. V., and Kubicek, C. P. 2011. Trichoderma: The genomics of opportunistic success. Nat. Rev. Microbiol. 9:749-759.

Duncan, K. E., and Howard, R. J. 2010. Biology of maize kernel infection by Fusarium verticillioides. Mol. Plant-Microbe Interact. 23:6-16.

Durgbanshi, A., Arbona, V., Pozo, O., Miersch, O., Sancho, J. V., and Gómez-Cadenas, A. 2005. Simultaneous determination of multiple phytohormones in plant extracts by liquid chromatography-electrospray tandem mass spectrometry. J. Agric. Food Chem. 53:8437-8442.

Eller, M. S., Holland, J. B., and Payne, G. A. 2008. Breeding for improved resistance to fumonisin contamination in maize. Toxin Rev. 27: 371-389.

Eulgem, T., and Somssich, I. E. 2007. Networks of WRKY transcription factors in defense signaling. Curr. Opin. Plant Biol. 10:366-371.

Ferrigo, D., Raiola, A., Piccolo, E., Scopel, C., and Causin, R. 2014 Trichoderma harzianum T22 induces in maize systemic resistance against Fusarium verticillioides. J. Plant Pathol. 96:133-142.

Folcher, L., Jarry, M., Weissenberger, A., Gerault, F., Eychenne, N., Delos, M., and Regnault-Roger, C. 2009. Comparative activity of agrochemical treatments on mycotoxin levels with regard to corn borers and Fusarium mycoflora in maize (Zea mays L.) fields. Crop Prot. 28:302-308.

Gao, Q. M., Kachroo, A., and Kachroo, P. 2014. Chemical inducers of systemic immunity in plants. J. Exp. Bot. 65:1849-1855.

Gas-Pascual, E., Berna, A., Bach, T. J., and Schaller, H. 2014. Plant oxidosqualene metabolism: Cycloartenol synthase-dependent sterol biosynthesis in Nicotiana benthamiana. PLoS One 9:e109156.

Glazebrook, J. 2005. Contrasting mechanisms of defense against biotrophic and necrotrophic pathogens. Annu. Rev. Phytopathol. 43:205-227.

Harman, G. E., Howell, C. R., Viterbo, A., Chet, I., and Lorito, M. 2004. Trichoderma species-Opportunistic, avirulent plant symbionts. Nat. Rev. Microbiol. 2:43-56.

He, K., Gou, X., Yuan, T., Lin, H., Asami, T., Yoshida, S., Russell, S. D., and Li, J. 2007. BAK1 and BKK1 regulate brassinosteroid-dependent growth and brassinosteroid-independent cell-death pathways. Curr. Biol. 17:1109-1115.

Hermosa, R., Rubio, M. B., Cardoza, R. E., Nicolás, C., Monte, E., and Gutiérrez, S. 2013. The contribution of Trichoderma to balancing the costs of plant growth and defense. Int. Microbiol. 16:69-80.

Hermosa, R., Viterbo, A., Chet, I., and Monte, E. 2012. Plant-beneficial effects of Trichoderma and of its genes. Microbiology 158:17-25.

Huot, B., Yao, J., Montgomery, B. L., and He, S. Y. 2014. Growth-defense tradeoffs in plants: A balancing act to optimize fitness. Mol. Plant 7: 1267-1287.

Iglesias, J., Presello, D. A., Botta, G., Lori, G. A., and Fauguel, C. M. 2010. Aggressiveness of Fusarium Section Liseola isolates causing maize ear rot in Argentina. J. Plant Pathol. 92:205-211.

Kazan, K., and Manners, J. M. 2009. Linking development to defense: Auxin in plant-pathogen interactions. Trends Plant Sci. 14:373-382.

Kersey, P. J., Allen, J. E., Armean, I., Boddu, S., Bolt, B. J., Carvalho-Silva, D., Christensen, M., Davis, P., Falin, L. J., Grabmueller, C., Humphrey, 
J., Kerhornou, A., Khobova, J., Aranganathan, N. K., Langridge, N., Lowy, E., McDowall, M. D., Maheswari, U., Nuhn, M., Ong, C. K., Overduin, B., Paulini, M., Pedro, H., Perry, E., Spudich, G., Tapanari, E., Walts, B., Williams, G., Tello-Ruiz, M., Stein, J., Wei, S., Ware, D., Bolser, D. M., Howe, K. L., Kulesha, E., Lawson, D., Maslen, G., and Staines, D. M. 2016. Ensembl Genomes 2016: More genomes, more complexity. Nucleic Acids Res. 44:D574-D580.

Kim, D., Pertea, G., Trapnell, C., Pimentel, H., Kelley, R., and Salzberg, S. L. 2013. TopHat2: Accurate alignment of transcriptomes in the presence of insertions, deletions and gene fusions. Genome Biol. 14: R36.

Koehler, B. 1942. Natural mode of entrance of fungi into corn ears and some symptoms that indicate infection. J. Agric. Res. 64:421-422.

Korolev, N., David, D. R., and Elad, Y. 2008. The role of phytohormones in basal resistance and Trichoderma-induced systemic resistance to Botrytis cinerea in Arabidopsis thaliana. BioControl 53:667-683.

Kumar, D. 2014. Salicylic acid signaling in disease resistance. Plant Sci. 228:127-134.

Lanubile, A., Bernardi, J., Battilani, P., Logrieco, A., and Marocco, A. 2012. Resistant and susceptible maize genotypes activate different transcriptional responses against Fusarium verticillioides. Physiol. Mol. Plant Pathol. 77:52-59.

Lanubile, A., Ferrarini, A., Maschietto, V., Delledonne, M., Marocco, A., and Bellin, D. 2014. Functional genomic analysis of constitutive and inducible defense responses to Fusarium verticillioides infection in maize genotypes with contrasting ear rot resistance. BMC Genomics 15:710.

Lanubile, A., Maschietto, V., Borrelli, V. M., Stagnati, L., Logrieco, A. F., and Marocco, A. 2017. Molecular basis of resistance to Fusarium ear rot in maize. Front. Plant Sci. 8:1774.

Lanubile, A., Pasini, L., Lo Pinto, M., Battilani, P., Prandini, A., and Marocco, A. 2011. Evaluation of broad spectrum sources of resistance to Fusarium verticillioides and advanced maize breeding lines. World Mycotoxin J. 4:43-51.

Liao, Y., Smyth, G. K., and Shi, W. 2014. featureCounts: An efficient general purpose program for assigning sequence reads to genomic features. Bioinformatics 30:923-930.

Logrieco, A., Mule, G., Moretti, A., and Bottalico, A. 2002. Toxigenic Fusarium species and mycotoxins associated with maize ear rot in Europe. Pages 597-609 in: Mycotoxins in Plant Disease. A. Logrieco, J. A. Bailey, L. Corazza, and B. M. Cooke, eds. Springer, Dordrecht, The Netherlands.

Love, M. I., Huber, W., and Anders, S. 2014. Moderated estimation of fold change and dispersion for RNA-seq data with DESeq2. Genome Biol. 15:550.

Mathys, J., De Cremer, K., Timmermans, P., Van Kerckhove, S., Lievens, B., Vanhaecke, M., Cammue, B. P., and De Coninck, B. 2012. Genomewide characterization of ISR induced in Arabidopsis thaliana by Trichoderma hamatum T382 against Botrytis cinerea infection. Front. Plant Sci. 3:108.

Mauch-Mani, B., Baccelli, I., Luna, E., and Flors, V. 2017. Defense priming: An adaptive part of induced resistance. Annu. Rev. Plant Biol. 68:485-512.

Munkvold, G. P. 2003. Cultural and genetic approaches to managing mycotoxins in maize. Annu. Rev. Phytopathol. 41:99-116.

Nawrocka, J., and Małolepsza, U. 2013. Diversity in plant systemic resistance induced by Trichoderma. Biol. Control 67:149-156.

Pandey, S. P., and Somssich, I. E. 2009. The role of WRKY transcription factors in plant immunity. Plant Physiol. 150:1648-1655.

Park, S.-W., Kaimoyo, E., Kumar, D., Mosher, S., and Klessig, D. F. 2007. Methyl salicylate is a critical mobile signal for plant systemic acquired resistance. Science 318:113-116.

Perazzolli, M., Moretto, M., Fontana, P., Ferrarini, A., Velasco, R., Moser, C., Delledonne, M., and Pertot, I. 2012. Downy mildew resistance induced by Trichoderma harzianum T39 in susceptible grapevines partially mimics transcriptional changes of resistant genotypes. BMC Genomics 13:660.

Pieterse, C. M., Van der Does, D., Zamioudis, C., Leon-Reyes, A., and Van Wees, S. C. 2012. Hormonal modulation of plant immunity. Annu. Rev. Cell Dev. Biol. 28:489-521.

Pieterse, C. M., Van Pelt, J. A., Ton, J., Parchmann, S., Mueller, M. J., Buchala, A. J., Métraux, J.-P., and Van Loon, L. C. 2000.
Rhizobacteria-mediated induced systemic resistance (ISR) in Arabidopsis requires sensitivity to jasmonate and ethylene but is not accompanied by an increase in their production. Physiol. Mol. Plant Pathol. 57:123-134.

Pieterse, C. M., Zamioudis, C., Berendsen, R. L., Weller, D. M., Van Wees, S. C., and Bakker, P. A. 2014. Induced systemic resistance by beneficial microbes. Annu. Rev. Phytopathol. 52:347-375.

Raguso, R. A. 2009. Floral scent in a whole-plant context: Moving beyond pollinator attraction. Funct. Ecol. 23:837-840.

Roux, M., Schwessinger, B., Albrecht, C., Chinchilla, D., Jones, A., Holton, N., Malinovsky, F. G., Tör, M., de Vries, S., and Zipfel, C. 2011. The Arabidopsis leucine-rich repeat receptor-like kinases BAK1/SERK3 and BKK1/SERK4 are required for innate immunity to hemibiotrophic and biotrophic pathogens. Plant Cell 23:2440-2455.

Saeed, A. I., Bhagabati, N. K., Braisted, J. C., Liang, W., Sharov, V., Howe, E. A., Li, J., Thiagarajan, M., White, J. A., and Quackenbush, J. 2006. TM4 microarray software suite. Methods Enzymol. 411: 134-193.

Salas-Marina, M. A., Silva-Flores, M. A., Uresti-Rivera, E. E., CastroLongoria, E., Herrera-Estrella, A., and Casas-Flores, S. 2011. Colonization of Arabidopsis roots by Trichoderma atroviride promotes growth and enhances systemic disease resistance through jasmonic acid/ethylene and salicylic acid pathways. Eur. J. Plant Pathol. 131: 15-26.

Saravanakumar, K., Fan, L., Fu, K., Yu, C., Wang, M., Xia, H., Sun, J., Li, Y., and Chen, J. 2016. Cellulase from Trichoderma harzianum interacts with roots and triggers induced systemic resistance to foliar disease in maize. Sci. Rep. 6: Article 35543.

Schaller, H. 2003. The role of sterols in plant growth and development. Prog. Lipid Res. 42:163-175.

Schlaeppi, K., and Bulgarelli, D. 2015. The plant microbiome at work. Mol. Plant-Microbe Interact. 28:212-217.

Segarra, G., Van der Ent, S., Trillas, I., and Pieterse, C. M. 2009. MYB72, a node of convergence in induced systemic resistance triggered by a fungal and a bacterial beneficial microbe. Plant Biol Stuttg 11:90-96.

Shoresh, M., Harman, G. E., and Mastouri, F. 2010. Induced systemic resistance and plant responses to fungal biocontrol agents. Annu. Rev. Phytopathol. 48:21-43.

Singh, K., Foley, R. C., and Oñate-Sánchez, L. 2002. Transcription factors in plant defense and stress responses. Curr. Opin. Plant Biol. 5: 430-436.

Tang, J., Han, Z., and Chai, J. 2016. Q\&A: What are brassinosteroids and how do they act in plants? BMC Biol. 14:113.

Thimm, O., Bläsing, O., Gibon, Y., Nagel, A., Meyer, S., Krüger, P., Selbig, J., Müller, L. A., Rhee, S. Y., and Stitt, M. 2004. MAPMAN: A userdriven tool to display genomics data sets onto diagrams of metabolic pathways and other biological processes. Plant J. 37:914-939.

Vargas, W. A., Martín, J. M. S., Rech, G. E., Rivera, L. P., Benito, E. P., Díaz-Mínguez, J. M., Thon, M. R., and Sukno, S. A. 2012. Plant defense mechanisms are activated during biotrophic and necrotrophic development of Colletotrichum graminicola in maize. Plant Physiol. 158: 1342-1358.

Wildermuth, M. C., Dewdney, J., Wu, G., and Ausubel, F. M. 2001. Isochorismate synthase is required to synthesize salicylic acid for plant defence. Nature 414:562-565.

Wrzaczek, M., Brosché, M., Salojärvi, J., Kangasjärvi, S., Idänheimo, N., Mersmann, S., Robatzek, S., Karpiński, S., Karpińska, B., and Kangasjärvi, J. 2010. Transcriptional regulation of the CRK/DUF26 group of receptorlike protein kinases by ozone and plant hormones in Arabidopsis. BMC Plant Biol. 10:95.

Yeh, Y.-H., Chang, Y.-H., Huang, P.-Y., Huang, J.-B., and Zimmerli, L. 2015. Enhanced Arabidopsis pattern-triggered immunity by overexpression of cysteine-rich receptor-like kinases. Front. Plant Sci. 6:322.

Zhang, X., Chen, S., and Mou, Z. 2010. Nuclear localization of NPR1 is required for regulation of salicylate tolerance, isochorismate synthase 1 expression and salicylate accumulation in Arabidopsis. J. Plant Physiol. 167:144-148.

\section{AUTHOR-RECOMMENDED INTERNET RESOURCE}

FastQC: http://www.bioinformatics.babraham.ac.uk/projects/fastqc/ 